\title{
Dietary fibre intake and its role in the oesophageal inflammation-metaplasia-adenocarcinoma sequence
}

\author{
H. G. Mulholland ${ }^{1}$, M. M. Cantwell ${ }^{1}$, L. A. Anderson ${ }^{1}$, B. T. Johnston ${ }^{2}$, R. G. P. Watson ${ }^{2}$, \\ S. J. Murphy ${ }^{3}$, H. R. Ferguson ${ }^{4}$, J. McGuigan ${ }^{2}$, J. V. Reynolds ${ }^{5}$, H. Comber ${ }^{6}$ and L. J. Murray ${ }^{1}$ \\ ${ }^{1}$ Cancer Epidemiology \& Prevention Research Group, Centre for Public Health, Queens University Belfast, \\ Belfast BT12 6BJ, UK, ${ }^{2}$ Belfast Health \& Social Care Trust, Belfast BT8 8BH, UK, ${ }^{3}$ Daisy Hill Hospital, Belfast BT35 8DR, \\ UK, ${ }^{4}$ Craigavon Area Hospital, Belfast BT63 5QQ, UK, ${ }^{5}$ St James's Hospital, Dublin, Republic of Ireland and \\ ${ }^{6}$ National Cancer Registry, Cork, Republic of Ireland
}

Recurrent gastric acid reflux can result in inflammation and mucosal erosions in the squamous epithelium of the oesophagus, a condition known as reflux oesophagitis (RO) ${ }^{(1)}$. Over time, Barrett's oesophagus (BO) can develop when these cells in the distal oesophagus undergo a permanent metaplastic change to columnar-type epithelium ${ }^{(2)}$. BO is a premalignant condition for oesophageal adenocarcinoma $(\mathrm{OAC})$ and incidence rates of this cancer have tripled in the UK over recent decades ${ }^{(2,3)}$. Foods containing dietary fibre may protect against oesophageal cancer $^{(4)}$; however, the association with OAC specifically, and the stage at which fibre may exert its effect in the inflammation-metaplasiaadenocarcinoma sequence, remains to be clarified.

In an all-Ireland case-control study information was collected from patients with OAC ( $n$ 224), long-segment BO ( $n$ 220), RO ( $n$ 219) and healthy population-based controls ( $n$ 256) on lifestyle factors and dietary intake using a 101-item FFQ between March 2002 and July 2005. Multiple logistic regression analysis was applied to examine the association between dietary Englyst fibre intake and disease risk, comparing the highest with the lowest tertiles of intake and also using continuous variables. Regression models adjusted for confounding variables including age, gender, energy intake, smoking, education, occupation, alcohol, medication use and after further adjustment for Helicobacter pylori infection.

No significant association was identified between fibre intake and RO risk. Dietary fibre was strongly inversely related to BO risk when examining both the highest $v$. lowest tertile of intake (OR 0.44 (95\% CI 0.25, 0.80)) and per $10 \mathrm{~g} / \mathrm{d}$ increment in intake (OR 0.54 (95\% CI $0.36,0.83)$ ). This protective association was slightly strengthened after further adjustment for $H$. pylori infection. An inverse association was also observed between fibre intake and OAC risk, although a dose-response relationship was not apparent, as the reduced risk peaked in the middle tertile of intake (OR 0.52 (95\% CI 0.29, 0.98)).

Fibre does not appear to have a role in the initiation stages of OAC development, as intake was unrelated to RO risk. The present study is the first to demonstrate a strong inverse association between fibre intake and BO risk, suggesting a protective effect of dietary fibre in the intermediate stage of the oesophageal inflammation-metaplasia-adenocarcinoma sequence. Fibre intake was also inversely associated with OAC risk, although the effect appeared to plateau in the middle tertile of intake.

1. Kahrilas PJ (1996) JAMA 276, 983-998.

2. Fitzgerald RC (2004) Aliment Pharmacol Ther 20, Suppl. 8, S45-S49.

3. Newnham A, Quinn MJ, Babb P et al. (2003) Aliment Pharmacol Ther 17, 665-676.

4. World Cancer Research Fund/American Institute for Cancer Research (2007) Food, Nutrition, Physical Activity, and the Prevention of Cancer: a Global Perspective. Washington, DC: AICR. 